\title{
Is the reaction between formic acid and protonated aminomethanol a possible source of glycine precursors in the interstellar medium?
}

\author{
Pilar Redondo, Antonio Largo, and Carmen Barrientos
}

\author{
Computational Chemistry Group, Departamento de Química Física y Química Inorgánica, Facultad de Ciencias, Universidad \\ de Valladolid, 47011 Valladolid, Spain \\ e-mail: [predondo; alargo; cbb]@qf.uva.es
}

Received 18 May 2015 / Accepted 28 May 2015

\begin{abstract}
Context. One of the most interesting questions in interstellar chemistry concerns whether we can detect the basic building blocks of proteins in astronomical sources. In ascertaining whether amino acids could be possible interstellar molecules, a crucial point is how they could be synthesized in the interstellar medium.

Aims. We do a theoretical study of the ion-molecule reaction involving protonated aminomethanol and formic acid to establish its viability in space. This ion-molecule reaction has been proposed by other authors as a possible way to produce glycine in the interstellar medium.

Methods. The relevant stationary points on the potential energy surface of the reaction between protonated aminomethanol and formic acid have been theoretically studied by using ab initio methods. The second-order Moller-Plesset level was employed, in conjunction with the correlation-consistent polarized valence triple-zeta (cc-pVTZ) basis set. In addition, the electronic energies were refined by means of single-point calculations at the $\operatorname{CCSD}(\mathrm{T})$ level (coupled cluster single and double excitation model augmented with a non-iterative treatment of triple excitations) on the MP2/cc-pVTZ geometries with the aug-cc-pVTZ basis set.

Results. Formation of protonated glycine is an exothermic process; however, the process presents a net activation barrier that makes this reaction unfeasible under interstellar conditions.

Conclusions. The reaction of protonated aminomethanol with formic acid does not seem to be a plausible source of interstellar glycine. This particular case is a clear example that a detailed study of the potential energy surface is needed to establish the relevance of a process in the interstellar medium.
\end{abstract}

Key words. astrobiology - astrochemistry - molecular processes - ISM: kinematics and dynamics - ISM: molecules - ISM: general

\section{Introduction}

Around 180 molecular species have been detected so far in the interstellar medium, and the list is increasing every year. These detections suggest a much richer chemistry than previously expected in the interstellar medium. One of the most complex questions in interstellar chemistry concerns the possible existence of bio-molecules. Undoubtedly, finding bio-molecules in the interstellar medium would have a deep impact on our vision of the origin of life. It is not surprising that so much effort has been devoted to finding amino acids, the basic building blocks of proteins, in astronomical sources. In particular, the simplest amino acid, glycine, has been searched for recursively, although with no success so far (Hollis et al. 1980; Snyder et al. 1983, 2005; Ceccarelli et al. 2000; Hollis et al. 2003a,b; Kuan et al. 2003; Jones et al. 2007; Cunningham et al. 2007).

Nevertheless, there is observational evidence of amino acids in extraterrestrial objects, such as meteorites (Cronin \& Pizarello 1997; Ehrenfreund et al. 2001; Glavin et al. 2010; Burton et al. 2013) or comets (Elsila et al. 2009). Furthermore, different experiments have shown the formation of amino acids after UV irradiation of interstellar ice analogues (Bernstein et al. 2002; Muñoz-Caro et al. 2002; Holtom et al. 2005; Elsila et al. 2007). Therefore, even though no conclusive detection of an amino acid in the interstellar medium has been reported, it is conceivable that they could be present in space. A recent three-phase (gas-phase, grain-surface, and bulk-ice) chemical model of hot cores (Garrod 2013) predicts that detection of glycine with instruments like ALMA that have greater sensitivity and spatial resolution could be plausible.

To ascertain whether amino acids could be possible interstellar molecules, a crucial point is how they could be synthesized in the interstellar medium. In view of the experimental results mentioned earlier, a possible way of obtaining amino acids is through reactions on the surface of interstellar dust particles. Different theoretical studies have been carried out on possible synthetic pathways taking place on the surface of interstellar grains leading to precursors of glycine (Woon 2001, 2002a,b; Rimola et al. 2010, 2012). In most of these studies, the reactions that were considered involved radical species.

In addition, amino acids could also be formed through gas phase reactions. Several proposals of different neutral-neutral reactions have been made (Hoyle 1976; Maeda \& Ohno 2004a,b, 2006; Basiuk \& Kobayashi 2004; Andreazza et al. 2006). In some cases theoretical studies have been carried out (Basiuk \& Bogillo 2000; Basiuk 2001) that conclude that these processes are not feasible under interstellar conditions. Ion-molecule reactions are more promising, since in many cases they are more likely to proceed without involving energy barriers. Different ion-molecule processes have been invoked as possible sources of glycine precursors. Experimental (Jackson et al. 2005a,b) and theoretical (Largo et al. 2004, 2008, 2010) studies of the 
reactions of ammonia cations with formic and acetic acids and of different neutrals with amines have been carried out. Some of these reactions involve significant barriers, whereas other barrier-free processes leading to precursors of glycine are less favorable than other competing channels.

Precursors of glycine and alanine were obtained in experiments involving ion-molecule reactions of hydroxylamine derivatives and carboxylic acids (Blagojevic et al. 2003; Snow et al. 2007). However, a recent theoretical study (Barrientos et al. 2012) has concluded that production of glycine from the reaction of either cationic or protonated hydroxylamine should be fairly inefficient under interstellar conditions, a result which agrees with a recently model developed by Garrod (2013). Therefore, until now there seems to be no successful proposal for a gas-phase reaction that could lead to precursors of interstellar glycine.

In the present work we perform a theoretical study of the viability of the ion-molecule reaction involving protonated aminomethanol and formic acid in space. This reaction has been proposed by other authors as one route toward producing glycine in the interstellar medium (Charnley 1999; Charnley et al. 2001; Kuan et al. 2003). However, to the best of our knowledge, this reaction has not been previously studied theoretically or experimentally. The paper is structured as follows. In the second section, we introduce the computational methods used in our study. Section 3 first presents the protonation of aminomethanol under interstellar conditions, and then the potential energy surface of the reaction of protonated aminomethanol with formic acid is analyzed. In the last section, we summarize the main findings of our study.

\section{Computational methods}

The characterization of stationary points on the potential energy surface for the reaction of aminomethanol with formic acid employed similar theoretical methods to our previous work on related systems (Barrientos et al. 2012; Largo et al. 2008, 2010), since these methods provide reliable enough results. Geometrical parameters of the different species were obtained at the second-order Moller-Plesset level (MP2) by employing the correlation-consistent polarized valence triple-zeta (cc-pVTZ) basis set developed by Dunning (1989) and Kendall et al. (1992). Harmonic vibrational frequencies were computed at the same level of theory as for the different optimized structures. This allows the zero-point vibrational energy correction (ZPE) to be estimated and the nature of the stationary points located on the potential energy surface to be confirmed, which is either a true minimum (all vibrational frequencies real) or a transition state (one of the frequencies, and just one, imaginary). Intrinsicreaction coordinate (IRC) calculations (Gonzalez \& Schelegel 1990; Gonzalez et al. 1989) were also carried out to check that the transition structures obtained correspond to the desired process.

Electronic energies were refined, over the MP2/cc-pVTZ geometries, through single-point calculations at the coupledcluster single and double excitation model augmented with a non-iterative triple excitation correction. This is usually referred to as CCSD(T) level (Raghavachari et al. 1989). In these calculations, the aug-cc-pVTZ basis set (Dunning 1989; Kendall et al. 1992), which also includes diffuse functions, was employed.

The same levels of theory were employed in the study of aminomethanol protonation but for these systems, given their size, the MP2 geometry optimization was also carried out with the aug-cc-pVTZ basis set. For these geometries singlepoint calculations at the $\operatorname{CCSD}(\mathrm{T})$ were performed with the aug-cc-pVQZ basis set (correlation-consistent polarized valence quadruple-zeta including diffuse functions Dunning 1989; Kendall et al. 1992). All calculations reported in this work were carried out with the Gaussian-09 program package (Frisch at al. 2010).

\section{Results and discussion}

Formic acid $(\mathrm{HCOOH})$ is a molecule present in the interstellar medium (Zuckerman et al. 1971; Winnewisser \& Churchwell 1975) that can evolve to more complex systems. Despite, aminomethanol $\left(\mathrm{NH}_{2} \mathrm{CH}_{2} \mathrm{OH}\right)$ is considered to be a key prebiotic interstellar molecule, it remains undetected in space, in part because of the difficulties synthethizing it in terrestrial laboratories, which makes it difficult to obtain a complete spectroscopic characterization. It has been predicted that aminomethanol is formed in interstellar ices through a radical-radical reaction between $\mathrm{NH}_{2}$ and $\mathrm{CH}_{2} \mathrm{OH}$, which are created in the photolysis of ammonia and methanol, respectively (Garrod et al. 2008). The experimental work of Bossa et al. (2009) shows that aminomethanol is formed from the thermal reaction of $\mathrm{NH}_{3}$ and $\mathrm{H}_{2} \mathrm{CO}$ in interstellar-ice analogues at low temperatures. They conclude that $\mathrm{NH}_{2} \mathrm{CH}_{2} \mathrm{OH}$ should be detectable in the gas phase in warm environments where the icy material is desorbed. From a theoretical point of view, inserting $\mathrm{O}\left({ }^{1} \mathrm{D}\right)$ into the $\mathrm{C}-\mathrm{H}$ bond of methyl amine gives aminomethanol as the most energetically favored pathway (Hays \& Widicus Weaver 2013). A simulation of the spectrum of aminomethanol based on its optimized geometry has also been reported. Feldmann et al. (2005) analyzed the stability of aminomethanol with respect to the loss of one water molecule and its conversion to methanimine. The calculated barrier height (about $55 \mathrm{kcal} / \mathrm{mol}$ ) indicates that aminomethanol is stable in relation to this process. Finally, the water-catalyzed reaction of formaldehyde and ammonia for generating $\mathrm{NH}_{2} \mathrm{CH}_{2} \mathrm{OH}$ has been also studied (Courmier et al. 2005).

\subsection{Protonation of aminomethanol}

Before analyzing the reaction of protonated aminomethanol with formic acid, we briefly discuss the protonation of aminomethanol. Proton transfer processes are common in the interstellar medium where $\mathrm{H}_{3}^{+}$is abundant. If aminomethanol is present in the gas phase, two different isomers could be obtained upon protonation: $\mathrm{NH}_{3} \mathrm{CH}_{2} \mathrm{OH}^{+}$if protonation takes place at the nitrogen atom, and $\mathrm{NH}_{2} \mathrm{CH}_{2} \mathrm{OH}_{2}^{+}$when the proton is attached to the oxygen atom.

The most relevant geometrical parameters for both isomers are collected in Fig. 1. Protonation of aminomethanol at the nitrogen atom is slightly more favorable than protonation at oxygen one, since $\mathrm{NH}_{3} \mathrm{CH}_{2} \mathrm{OH}^{+}$is located $0.96 \mathrm{kcal} / \mathrm{mol}$ lower in energy than $\mathrm{NH}_{2} \mathrm{CH}_{2} \mathrm{OH}_{2}^{+}$at the $\operatorname{CCSD}(\mathrm{T}) /$ aug-cc-pVQZ//MP2/aug-cc-pVTZ level. Proton affinities for aminomethanol are shown in Table 1. Considering the similar stability of both protonated isomers, the proton affinities are close for protonation at either a nitrogen or an oxygen atom. In both cases, proton affinities are relatively high if comparison is made to the values of some abundant interstellar molecules. For example, the proton affinities of $\mathrm{H}_{2}, \mathrm{CO}$, and, $\mathrm{C}_{2} \mathrm{H}_{2}$ are 100,143 , and $152 \mathrm{kcal} / \mathrm{mol}$, respectively. Therefore $\mathrm{NH}_{2} \mathrm{CH}_{2} \mathrm{OH}$ should react quite easily in proton-rich interstellar media to give the protonated species. 
P. Redondo et al.: The reaction between formic acid and protonated aminomethanol

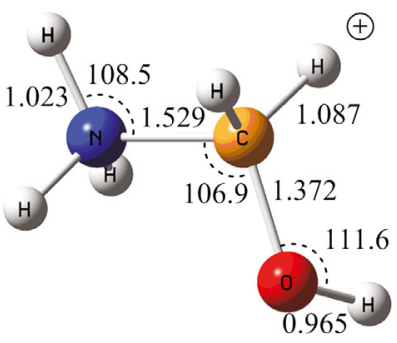

$\mathrm{NH}_{3} \mathrm{CH}_{2} \mathrm{OH}^{+}\left({ }^{1} \mathrm{~A}\right)$

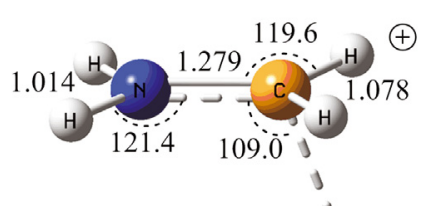

$2.451^{3}$

$\mathrm{NH}_{2} \mathrm{CH}_{2} \mathrm{OH}_{2}^{+}\left({ }^{1} \mathrm{~A}^{\prime}\right)$

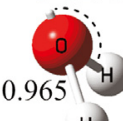

Fig. 1. MP2/aug-cc-pVTZ optimized geometries for the $\mathrm{NH}_{3} \mathrm{CH}_{2} \mathrm{OH}^{+}$ and $\mathrm{NH}_{2} \mathrm{CH}_{2} \mathrm{OH}_{2}^{+}$isomers. Distances are given in angstroms and angles in degrees.

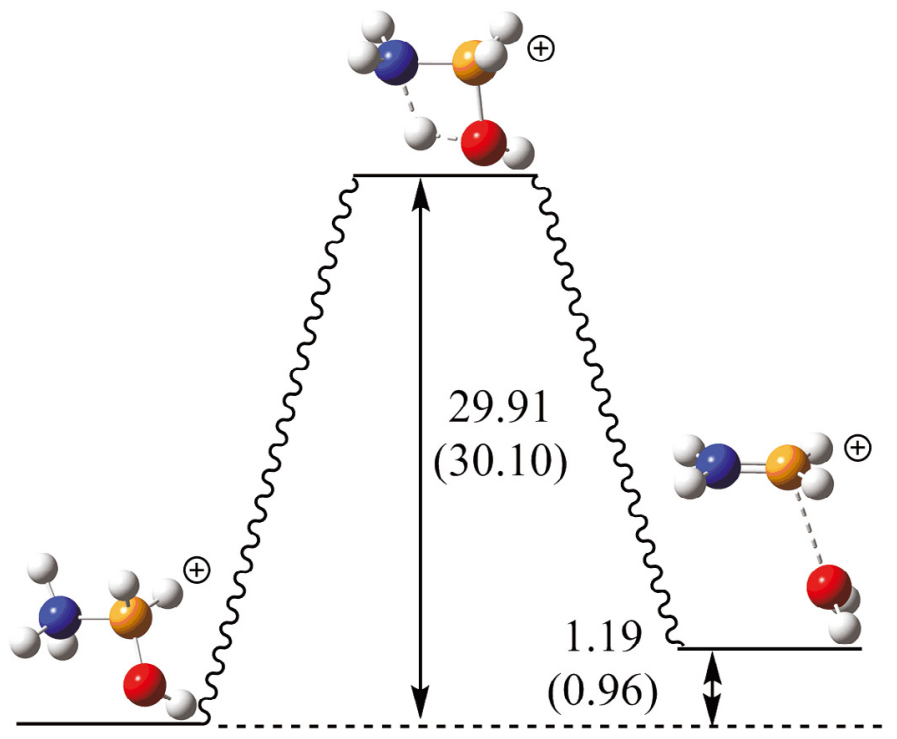

Fig. 2. Energy profile in $\mathrm{kcal} / \mathrm{mol}$ for the isomerization of protonated aminomethanol. Relative energies are calculated with respect to N-protonated aminomethanol at the CCSD(T)/aug-cc-pVTZ//MP2/ccpVTZ and $\operatorname{CCSD}(\mathrm{T}) /$ aug-cc-pVQZ//MP2/aug-cc-pVTZ (in parentheses) levels.

We have also considered the isomerization process between both isomers of protonated aminomethanol, and the results are summarized in Fig. 2. The conversion barrier of N-protonated into O-protonated aminomethanol is $30.10 \mathrm{kcal} / \mathrm{mol}$ (corresponding to a temperature of $15100 \mathrm{~K}$ ) at the $\operatorname{CCSD}(\mathrm{T}) / \mathrm{aug}-$ cc-pVQZ//MP2/aug-cc-pVTZ level. Consequently, the two protonated forms may coexist in the interstellar medium, and both isomers could be considered as reactants. A similar situation is reported by Snow et al. (2007) in the case of hydroxylamine.

It can be seen from Table 1 and Fig. 2 that similar results are obtained at both the $\operatorname{CCSD}(\mathrm{T}) /$ aug-cc-pVQZ//MP2/augcc-pVTZ and CCSD(T)/aug-cc-pVTZ//MP2/cc-pVTZ levels. Energy differences are in all cases less than $1 \mathrm{kcal} / \mathrm{mol}$; therefore, the second approach seems to be reliable in the study of this kind of system and is employed for characterizing stationary points on the potential energy surface of the reaction between protonated aminomethanol and formic acid.

\subsection{Reaction of protonated aminomethanol and formic acid}

In this section we report the theoretical results for the ionmolecule reaction of protonated aminomethanol and formic acid.
Table 1. Proton affinities (PA) in $\mathrm{kcal} / \mathrm{mol}$ for aminomethanol at $298 \mathrm{~K}$ calculated at different levels of theory.

\begin{tabular}{lcc}
\hline \hline Level & $\mathrm{PA}(\mathrm{N})$ & $\mathrm{PA}(\mathrm{O})$ \\
\hline CCSD(T)/aug-cc-pVQZ// & & \\
MP2/aug-cc-pVTZ & 210.31 & 209.35 \\
CCSD(T)/aug-cc-pVTZ// & & \\
MP2/cc-pVTZ & 210.58 & 209.39 \\
\hline
\end{tabular}

Table 2. Relative energies (in $\mathrm{kcal} / \mathrm{mol}$ ) with respect to reactants at the MP2/cc-pVTZ//MP2/cc-pVTZ ( $\triangle \mathrm{E}$ MP2) and CCSD(T)/aug-cc$\mathrm{pVTZ} / / \mathrm{MP} 2 / \mathrm{cc}-\mathrm{pVTZ}(\triangle \mathrm{E} \mathrm{CCSD}(\mathrm{T}))$ levels of theory for the possible products of the reaction between protonated aminomethanol and formic acid.

\begin{tabular}{lcc}
\hline \hline & $\Delta E$ & $\Delta E$ \\
& $\mathrm{MP} 2$ & $\mathrm{CCSD}(\mathrm{T})$ \\
\hline Reactants & & \\
$\mathrm{HCOOH}\left({ }^{1} \mathrm{~A}^{\prime}\right)+\mathrm{NH}_{3} \mathrm{CH}_{2} \mathrm{OH}^{+}\left({ }^{1} \mathrm{~A}\right)$ & 0.00 & 0.00 \\
$\mathrm{HCOOH}\left({ }^{1} \mathrm{~A}^{\prime}\right)+\mathrm{NH}_{2} \mathrm{CH}_{2} \mathrm{OH}_{2}^{+}\left({ }^{1} \mathrm{~A}^{\prime}\right)$ & 2.64 & 1.19 \\
Products & & \\
$\mathrm{HC}(\mathrm{OH})+\left({ }^{1} \mathrm{~A}^{\prime}\right)+\mathrm{NH}_{2} \mathrm{CH}_{2} \mathrm{OH}\left({ }^{1} \mathrm{~A}^{\prime}\right)$ & 33.95 & 32.83 \\
$\mathrm{NH}_{3} \mathrm{CH}_{2} \mathrm{COOH}\left({ }^{1} \mathrm{~A}_{1}\right)(\mathrm{pg} 1)+\mathrm{H}_{2} \mathrm{O}\left({ }^{1} \mathrm{~A}_{1}\right)$ & -16.38 & -16.46 \\
$\left.\mathrm{NH}_{2} \mathrm{CHCH} \mathrm{OH}\right)_{2}^{+}\left({ }^{1} \mathrm{~A}\right)(\mathrm{pg} 2)+\mathrm{H}_{2} \mathrm{O}\left({ }^{1} \mathrm{~A}_{1}\right)$ & 8.20 & 4.87 \\
$\mathrm{NH}_{2} \mathrm{CH}_{2} \mathrm{C}(\mathrm{OH})_{2}^{+}\left({ }^{1} \mathrm{~A}\right)(\mathrm{pg} 3)+\mathrm{H}_{2} \mathrm{O}\left({ }^{1} \mathrm{~A}_{1}\right)$ & 13.15 & 11.32 \\
$\mathrm{NH}_{3} \mathrm{CHOCHOH}^{+}\left({ }^{1} \mathrm{~A}\right)(\mathrm{pg} 4)+\mathrm{H}_{2} \mathrm{O}\left({ }^{1} \mathrm{~A}_{1}\right)$ & 22.68 & 22.17 \\
$\mathrm{OHCH}_{2} \mathrm{C}(\mathrm{OH})_{2}^{+}\left({ }^{1} \mathrm{~A}\right)+\mathrm{NH}_{3}\left({ }^{1} \mathrm{~A}_{1}\right)$ & 9.09 & 7.92 \\
\hline
\end{tabular}

Notes. Zero-point vibrational energy differences have been included.

Table 2 summarizes the calculated reaction energies for different products. We have included only those products that were reached from the reactants.

From a qualitative point of view, the MP2 and CCSD(T) values shown in Table 2 are rather similar. First, we can point out that proton transfer from protonated aminomethanol to $\mathrm{HCOOH}$ is endothermic ( $\Delta E=32.83 \mathrm{kcal} / \mathrm{mol}$ at the $\operatorname{CCSD}(\mathrm{T})$ level). Therefore, simple proton transfer is not a viable process, and only transformation reactions of formic acid into more complex systems could be feasible processes in the gas phase. One possible product for the reaction between protonated aminomethanol and formic acid is the formation of protonated glycine and elimination of a water molecule. As seen in Table 2, only formation of $\mathrm{N}$-protonated glycine $\left(\mathrm{NH}_{3} \mathrm{CH}_{2} \mathrm{COOH}^{+}(\mathrm{pg} 1)\right)$, the most stable isomer of protonated glycine (Lattelais et al. 2011), is an exothermic process $(\Delta E=-16.46 \mathrm{kcal} / \mathrm{mol}$ at the $\operatorname{CCSD}(\mathrm{T})$ level). O-protonated glycine $\mathrm{NH}_{2} \mathrm{CH}_{2} \mathrm{C}(\mathrm{OH})_{2}^{+}(\mathrm{pg} 3)$ is located $11.32 \mathrm{kcal} / \mathrm{mol}$ above the most stable reactants at the CCSD(T) level, which is an endothermic process. Formation of other protonated isomers of glycine (isomers pg2 and pg4) are also endothermic processes. Protonated glycolic acid, $\mathrm{OHCH}_{2} \mathrm{C}(\mathrm{OH})_{2}^{+}$, can be obtained from the title reaction by the elimination of a molecule of ammonia, but this product is located $7.92 \mathrm{kcal} / \mathrm{mol}$ above the most stable reactants. We have seen in the previous section that the energy difference between both isomers of protonated aminometanol is quite small (about $1 \mathrm{kcal} / \mathrm{mol}$ ), so the viability of the different processes is the same when both are considered as reactants. We can conclude that from a thermodynamic point of view, only formation of $\mathrm{N}$-protonated glycine is viable in space conditions.

To assess the role of these reactions in the formation of protonated glycine in the interstellar medium, a detailed study of the corresponding potential energy surface is necessary. This allows 
us to know if a process is barrier free and, therefore, viable from a kinetic point of view. It should be pointed out that even if the reaction between formic acid and protonated aminomethanol is barrierless, additional considerations are needed to establish that this reaction could play an important role in the synthesis of glycine. In this way, a key factor is knowing the branching ratio leading from protonated glycine to glycine and hydrogen atom in the dissociative recombination reaction of protonated glycine with free electrons. An example of the relevance of determining branching ratios is given in the study of the dissociative recombination of protonated methanol (Geppert et al. 2006). We searched for possible intermediates for the reactions of $\mathrm{NH}_{3} \mathrm{CH}_{2} \mathrm{OH}^{+}$and $\mathrm{NH}_{2} \mathrm{CH}_{2} \mathrm{OH}_{2}^{+}$with formic acid, and the corresponding transition states connecting these intermediates have also been characterized. All intermediates considered in the present work have vibrational frequencies that are real, thus corresponding to true minima on the potential energy surface. On the other hand, all transition states have just one imaginary frequency that is associated to the corresponding reaction coordinate in each case. In addition, IRC calculations confirm a correct connection. When we take the multiplicity of reactants into account, the reaction takes place on the singlet potential energy surface. The energy profile for the reaction $\mathrm{NH}_{3} \mathrm{CH}_{2} \mathrm{COOH}^{+}\left(\mathrm{NH}_{2} \mathrm{CH}_{2} \mathrm{C}(\mathrm{OH})_{2}^{+}\right)+\mathrm{HCOOH}$ is shown in Fig. 3. The energy values reported in Fig. 3 correspond to the CCSD(T)/aug-cc-pVTZ//MP2/cc-pVTZ level.

The reaction of $\mathrm{N}$-protonated aminomethanol with $\mathrm{HCOOH}$ starts with the formation of an ion-molecule complex $\mathrm{I} 1\left({ }^{1} \mathrm{~A}\right)$ obtained through the interaction between one of the hydrogen atoms bonded to nitrogen in $\mathrm{NH}_{3} \mathrm{CH}_{2} \mathrm{OH}^{+}$with the carbonyl oxygen of formic acid. This intermediate is located $-18.80 \mathrm{kcal} / \mathrm{mol}$ below the reactants at the $\operatorname{CCSD}(\mathrm{T}) /$ aug-ccpVTZ level. Hydrogen-atom migration from $\mathrm{CH}_{2}$ to $\mathrm{OH}$ gives $\mathrm{I} 2\left({ }^{1} \mathrm{~A}\right)$ that involves transition state $\mathrm{TS} 1\left({ }^{1} \mathrm{~A}\right)$. This intermediate evolves to $\mathrm{I} 3\left({ }^{1} \mathrm{~A}\right)$ through transition state $\operatorname{TS} 2\left({ }^{1} \mathrm{~A}\right)$, where a proton transfer to carbonyl oxygen and simultaneous formation of a C-C bond take place. As can be seen in Fig. 3, both transition states, $\operatorname{TS} 1\left({ }^{1} \mathrm{~A}\right)$ and $\operatorname{TS} 2\left({ }^{1} \mathrm{~A}\right)$, and intermediate $\mathrm{I} 2\left({ }^{1} \mathrm{~A}\right)$ are located clearly above the reactants $(64.10 \mathrm{kcal} / \mathrm{mol}$, $50.84 \mathrm{kcal} / \mathrm{mol}$, and $45.65 \mathrm{kcal} / \mathrm{mol}$ at the $\operatorname{CCSD}(\mathrm{T}) / \mathrm{aug}-\mathrm{cc}-$ pVTZ level, respectively), whereas intermediate $\mathrm{I} 3\left({ }^{1} \mathrm{~A}\right)$ is located $11.47 \mathrm{kcal} / \mathrm{mol}$ below the reactants at the same level. We have found that both carbon atoms should be activated for a $\mathrm{C}-\mathrm{C}$ bond to be formed. These activation processes involve a clear energy barrier (above $32000 \mathrm{~K}$ ). Intermediate I3 $\left({ }^{1} \mathrm{~A}\right.$ ) could evolve protonated glycine through path $a .1$, which can be summarized as

$$
\begin{aligned}
& \mathrm{NH}_{3} \mathrm{CH}_{2} \mathrm{OH}^{+}\left({ }^{1} \mathrm{~A}\right)+\mathrm{HCOOH}\left({ }^{1} \mathrm{~A}^{\prime}\right) \longrightarrow \mathrm{I} 1\left({ }^{1} \mathrm{~A}\right) \longrightarrow \mathrm{TS} 1\left({ }^{1} \mathrm{~A}\right) \\
& \longrightarrow \mathrm{I} 2\left({ }^{1} \mathrm{~A}\right) \longrightarrow \mathrm{TS} 2\left({ }^{1} \mathrm{~A}\right) \longrightarrow \mathrm{I} 3\left({ }^{1} \mathrm{~A}\right) \longrightarrow \mathrm{TS} 3\left({ }^{1} \mathrm{~A}\right) \longrightarrow \mathrm{I} 4\left({ }^{1} \mathrm{~A}\right) \\
& \longrightarrow \mathrm{TS} 4\left({ }^{1} \mathrm{~A}\right) \longrightarrow \mathrm{NH}_{3} \mathrm{CH}_{2} \mathrm{COOH}^{+}\left({ }^{1} \mathrm{~A}^{\prime}\right)+\mathrm{H}_{2} \mathrm{O}\left({ }^{1} \mathrm{~A}_{1}\right) \text { Path a.l. }
\end{aligned}
$$

Once $\mathrm{I} 3\left({ }^{1} \mathrm{~A}\right)$ is reached, the simultaneous migration of both a hydrogen atom and the $\mathrm{NH}_{3}$ group through transition state $\operatorname{TS} 3\left({ }^{1} \mathrm{~A}\right)$ leads to intermediate $\mathrm{I} 4\left({ }^{1} \mathrm{~A}\right)$ located $-13.60 \mathrm{kcal} / \mathrm{mol}$ below the reactants at the $\operatorname{CCSD}(\mathrm{T}) /$ aug-cc-pVTZ level. The elimination of one water molecule and subsequent migration of $\mathrm{NH}_{3}$ group involving transition state $\mathrm{TS} 4\left({ }^{1} \mathrm{~A}\right)$ give the most stable isomer of protonated glycine (pg1). Transition states $\operatorname{TS} 3\left({ }^{1} \mathrm{~A}\right)$ and TS4 $\left({ }^{1} \mathrm{~A}\right.$ ) are clearly located above the reactants (see Fig. 3 ). Even if the process described in path a.1 is clearly exothermic, it implies a net activation barrier, so it is not viable under interstellar conditions.
The loss of a $\mathrm{NH}_{3}$ molecule from intermediate $\mathrm{I} 4\left({ }^{1} \mathrm{~A}\right)$ leads to protonated glycolic acid (path a.2), which is an endothermic product. From this intermediate, this process follows the same steps as path a.1. It is therefore not allowed in space because it implies significant energy barriers.

Other possible evolution paths have been considered from intermediate I3 $\left({ }^{1} \mathrm{~A}\right)$. Considering this intermediate, the migration of a hydrogen atom from nitrogen to oxygen through transition state TS5 $\left({ }^{1} \mathrm{~A}\right)$ leads to intermediate $\mathrm{I} 5\left({ }^{1} \mathrm{~A}\right)$. From I5 $\left({ }^{1} \mathrm{~A}\right)$, two different isomers of protonated glycine (pg2 and pg3) could be reached through paths $b .1$ and $b .2$. In both cases, the full process is endothermic and presents a high energy barrier. Finally, the loss of a $\mathrm{H}_{2} \mathrm{O}$ molecule from $\mathrm{I} 3\left({ }^{1} \mathrm{~A}\right)$ gives another isomer of protonated glycine (pg4) involving transition state $\operatorname{TS} 7\left({ }^{1} \mathrm{~A}\right)$ (path c). This path is clearly not viable under interstellar conditions given its endothermicity and high energy barrier.

When we start the reaction by considering the less stable isomer of protonated aminomethanol, the direct interaction of $\mathrm{NH}_{2} \mathrm{CH}_{2} \mathrm{OH}_{2}^{+}$with formic acid gives the ion-molecule intermediate $\mathrm{I} 7\left({ }^{1} \mathrm{~A}\right)$, which is located $10.19 \mathrm{kcal} / \mathrm{mol}$ below the reactants at the CCSD(T)/aug-cc-pVTZ level. As can be seen in Fig. 3, the initial ion-molecule complex is more stable when the reaction is initiated by $\mathrm{N}$-protonated aminomethanol (about $8.61 \mathrm{kcal} / \mathrm{mol}$ at the $\operatorname{CCSD}(\mathrm{T}) /$ aug-cc-pVTZ level). From intermediate $\mathrm{I} 7\left({ }^{1} \mathrm{~A}\right)$, the activation of the carbon atom in the $\mathrm{NH}_{2} \mathrm{CH}_{2} \mathrm{OH}_{2}^{+}$fragment takes place through transition state $\operatorname{TS} 8\left({ }^{1} \mathrm{~A}\right)$ where one hydrogen atom migrates from a carbon atom to a nitrogen one, reaching the same intermediate that in the case of $\mathrm{N}$-protonated aminomethanol, I2 $\left({ }^{1} \mathrm{~A}\right)$, path $d$ :

$$
\begin{aligned}
& \mathrm{NH}_{2} \mathrm{CH}_{2} \mathrm{C}(\mathrm{OH})_{2}^{+}\left({ }^{1} \mathrm{~A}^{\prime}\right)+\operatorname{HCOOH}\left({ }^{1} \mathrm{~A}^{\prime}\right) \longrightarrow \mathrm{I} 7\left({ }^{1} \mathrm{~A}\right) \longrightarrow \\
& \mathrm{TS} 8\left({ }^{1} \mathrm{~A}\right) \longrightarrow \mathrm{I} 2\left({ }^{1} \mathrm{~A}\right) \longrightarrow \ldots \text { Path } d .
\end{aligned}
$$

Transition state $\operatorname{TS} 8\left({ }^{1} \mathrm{~A}\right)$ is clearly located above the reactants $(73.76 \mathrm{kcal} / \mathrm{mol}$ or $37100 \mathrm{~K}$ at the $\mathrm{CCSD}(\mathrm{T}) /$ aug-cc-pVTZ level), therefore subsequent processes imply a net activation barrier and are not viable under interstellar conditions.

From the analysis of the potential energy surface of the reaction between both isomers of protonated aminomethanol and formic acid, we notice that even if formation of the most stable isomer of protonated glycine (pg1) is an exothermic process, a net activation barrier is found in the paths that lead to this product.

\section{Conclusions}

We have carried out a theoretical study of the reaction between protonated aminomethanol and formic acid. The different intermediate species, as well as the most relevant transition states for these reactions, were characterized. In addition, we considered the protonation of aminomethanol.

Proton affinities for the protonation of aminomethanol either in a nitrogen or an oxygen atom are relatively high (210.31 and $209.35 \mathrm{kcal} / \mathrm{mol}$ at the CCSD(T)/aug-cc-pVQZ level, respectively), therefore $\mathrm{NH}_{2} \mathrm{CH}_{2} \mathrm{OH}$ should react quite easily in proton-rich interstellar media to yield the protonated species. The conversion barrier of $\mathrm{N}$-protonated into O-protonated aminomethanol is calculated to be $15100 \mathrm{~K}$ at the CCSD(T)/aug-cc-pVQZ level. Consequently, the two protonated forms may coexist in the interstellar medium, and both isomers could be considered as reactants.

A priori, and just from a thermodynamic analysis, the reaction between protonated aminomethanol and formic acid 
P. Redondo et al.: The reaction between formic acid and protonated aminomethanol

Path a.2

Path b. 1

Path b.2

Path c

Path d
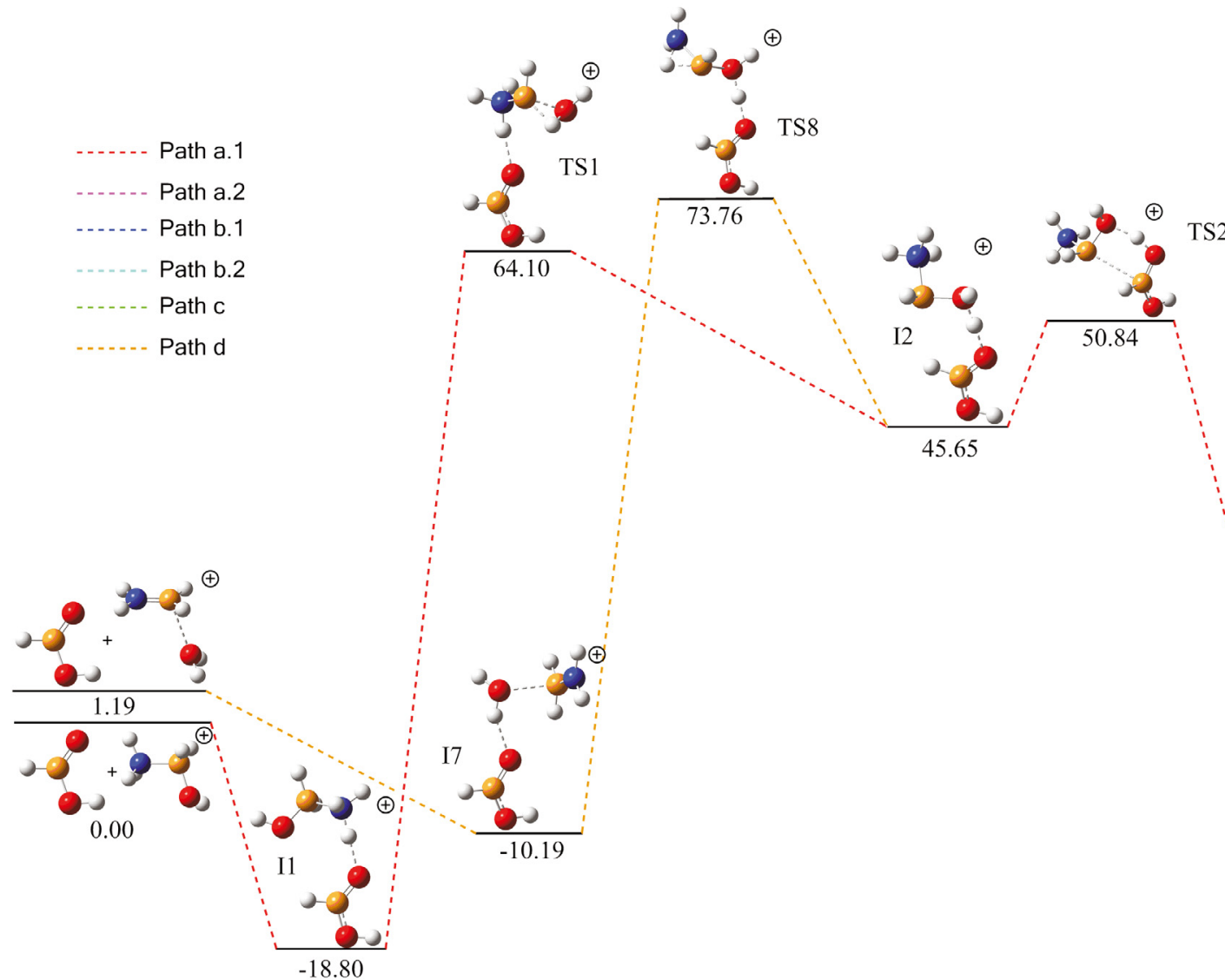

I7
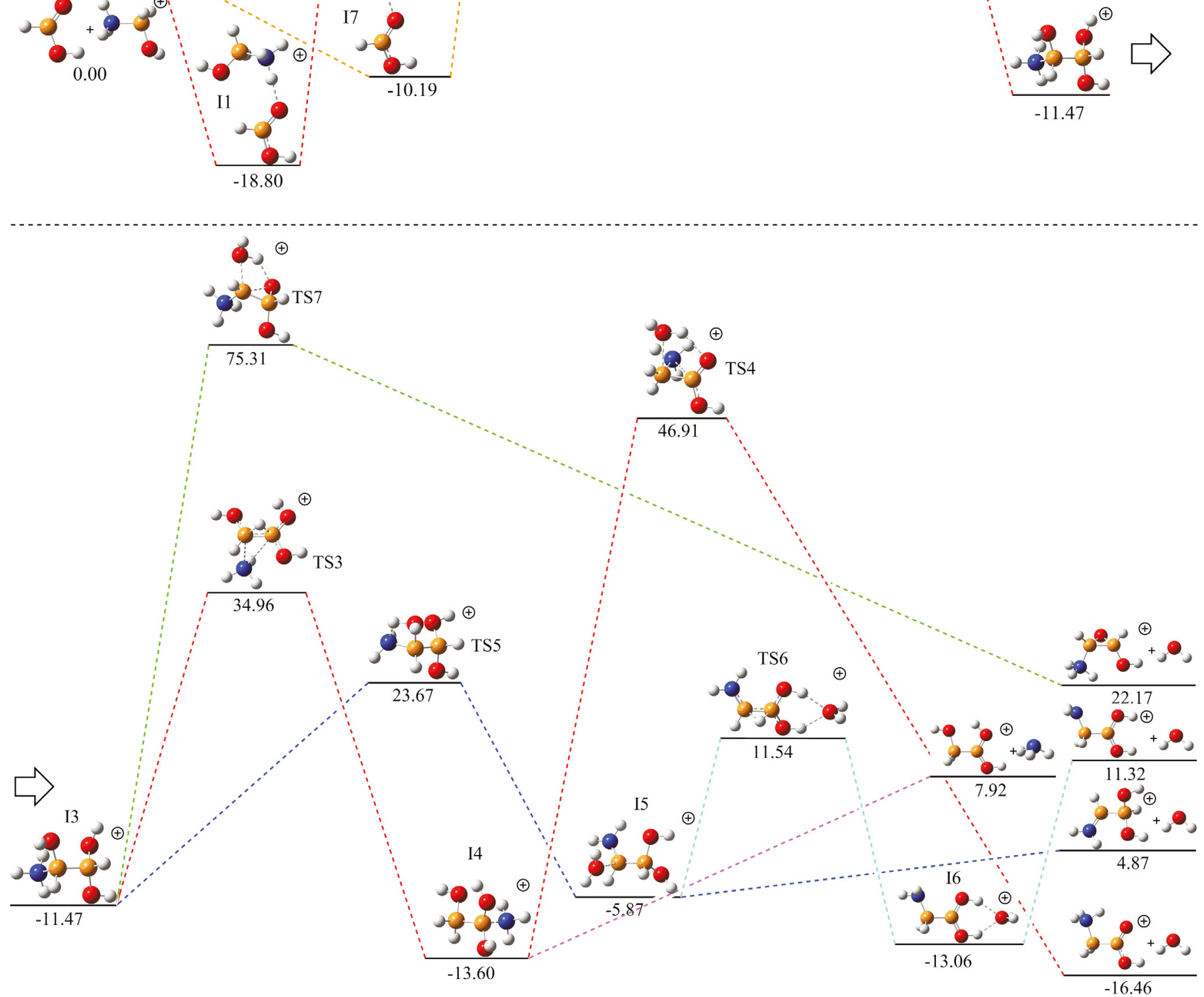

Fig. 3. Energy profile in $\mathrm{kcal} / \mathrm{mol}$ for the reaction of $\mathrm{NH}_{3} \mathrm{CH}_{2} \mathrm{OH}^{+}\left(\mathrm{NH}_{2} \mathrm{CH}_{2} \mathrm{OH}_{2}^{+}\right)$with $\mathrm{HCOOH}$ at the $\mathrm{CCSD}(\mathrm{T}) / \mathrm{aug}$-cc-pVTZ//MP2/ccpVTZ level (including zero-point vibrational energy differences). 
to give N-protonated glycine (pg1) should be feasible under interstellar conditions, since it is an exothermic process $(\Delta E=-16.46 \mathrm{kcal} / \mathrm{mol}$ at the $\operatorname{CCSD}(\mathrm{T}) /$ aug-cc-pVTZ level). A detailed study of the corresponding potential energy surface was reported to explore the viability of these reactions in the interstellar medium and their possible role in forming protonated glycine in space. From the analysis of the potential energy surface we can conclude that even if formation of the most stable isomer of protonated glycine is an exothermic process, a significant net activation barrier (about $64 \mathrm{kcal} / \mathrm{mol}$ at the CCSD(T)/aug-cc-pVTZ level corresponding to a temperature of $32000 \mathrm{~K}$ ) is found in the paths leading to this product.

Therefore, we conclude that the reaction of protonated aminomethanol with formic acid, which has been invoked as a possible source of interstellar glycine, does not seem viable in the interstellar medium. This particular case is a clear example that a theoretical detailed study of the potential energy surface is needed to establish the relevance of any process in the interstellar medium.

Acknowledgements. This research has been supported by the Junta de Castilla y León (Spain; Grant No. VA077U13).

\section{References}

Andreazza, H. J., Fitzgerald, M., \& Bowie, J. H. 2006, Org. Biomol. Chem., 4, 2466

Barrientos, C., Redondo, P., Largo, L., Rayon, V. M., \& Largo, A. 2012, ApJ, 748, 99

Basiuk, V. A. 2001, J. Phys. Chem. A, 105, 4252

Basiuk V. A., \& Bogillo, V. I. 2000, Adv. Space Res., 30, 1439

Basiuk, V. A., \& Kobayashi, Y. 2004, Int. J. Quantum Chem., 99, 92

Bernstein, M. P., Dworkin, J. P., Sandford, S. A., Cooper, G. W., \& Allamandola, L. J. 2002, Nature, 416, 401

Blagojevic, V., Petrie, S., \& Bohme, D. K. 2003, MNRAS, 339, L7

Bossa, J. B., Theule, P., Duvernay, F., \& Chiavassa, T. 2009, ApJ, 707, 1524

Burton, A. S., Elsila, J. E., Hein, J. E., et al. 2013, Meteorit. Planet. Sci., 48, 390

Ceccarelli, C., Loinard, L., Castets, A., Faure, A., \& Leffloch, B. 2000, A\&A, 362,1122

Charnley, S. B. 1999, in The Bridge Between the Big Bang and Biology, ed. F. Giovannelli (Rome: CNR), 139

Charnley, S. B., Ehrenfreund, P., \& Kuan, Y.-J. 2001, Spectrochim. Acta A, 57, 685

Courmier, D., Gardebien, F., Minot, C., \& St.-Amant, A. 2005, Chem. Phys. Lett., 405, 357

Cronin, J. R., \& Pizzarello, S. 1997, Science, 275, 951

Cunningham, M. R., Jones, P. A., Godfrey, P. D., et al. 2007, MNRAS, 376, 1201

Dunning, T. H. 1989, J. Chem. Phys., 90, 1007

Ehrenfreund, P., Glavin, D. P., Botta, O., Cooper, G., \& Bada, J. L. 2001, PNAS, 98, 2138

Elsila, J. E., Dworkin, J. P., Bernstein, M. P., Martin, M. P., \& Sandford, S. A. 2007, ApJ, 660, 911
Elsila, J. E., Glavin, D. P., \& Dworkin, J. P. 2009, Meteorit. Planet. Sci., 44, 1323 Feldmann, M. T., Widicus S. L., Blake, G. A., Kent IV, D. R., \& Goddard III, W. A. 2005, J. Chem. Phys., 123, 034304

Frisch, M. J., Trucks, G. W., Schlegel, H. B., et al. 2010, Gaussian 09, Gaussian Inc, Wallingford CT

Garrod, R. T. 2013, ApJ, 765, 60

Garrod, R. T., Widicus Weaver, S. L., \& Herbst, E. 2008, ApJ, 682, 283

Geppert, W. D., Hamberg, M., Thomas, R. D., et al. 2006, Faraday Discuss., 133, 177

Glavin, D. P., Aubrey, A. D., Callahan, M. P., et al. 2010, Meteorit. Planet. Sci, 45,1695

Gonzalez, C., \& Schelegel, H. B. 1989, J. Chem. Phys., 90, 2154

Gonzalez, C., \& Schelegel, H. B. 1990, J. Phys. Chem., 94, 5523

Hays, B. M., \& Widicus Weaver, S. L. 2013, J. Phys. Chem. A, 117, 7142

Hollis, J. M., Snyder, L. E., Suenram, R. D., \& Lovas, F. J. 1980, ApJ, 241, 1001

Hollis, J. M., Pedelty, J. A., Boboltz, D. A., et al. 2003a, ApJ, 596, L235

Hollis, J. M., Pedelty, J. A., Snyder, L. E., et al. 2003b, ApJ, 588, 353

Holtom, P. D., Bennett, C. J., Osamura, Y., Mason, N. J., \& Kaiser, R. I. 2005, ApJ, 626, 940

Hoyle F., \& Wickramasinghe, N. C. 1976, Nature, 264, 45

Jackson, D. M., Stibrich, N. J., Adams, N. G., \& Babcock, L. M. 2005a, Int. Mass. Spectrom., 243, 115

Jackson, D. M., Stibrich, N. J., McLain, J. L., et al. 2005b, Int. Mass. Spectrom., 247,55

Jones, P. A., Cunningham, M. R., Godfrey, P. D., \& Cragg, D. M. 2007, MNRAS, 374,579

Kendall, R. A., Dunning, T. H., \& Harrison, R. J. 1992, J. Chem. Phys., 96, 6796

Kuan, Y.-J., Charnley, S. B., Huang, H-C., Tseng W-L., \& Kisiel, Z. 2003, ApJ, 593,848

Largo, A., Redondo, P. \& Barrientos, C. 2004, Int. J. Quantum Chem., 98, 355

Largo, L., Rayón, V. M., Barrientos, C., Largo A., \& Redondo P. 2008, J. Chem. Theory Comput., 4, 2085

Largo, L., Redondo, P., Rayón, V. M., Largo, A., \& Barrientos, C. 2010, A\&A, 516, A79

Lattelais, M., Pauzat, F., Pilme, J., Ellinger, Y., \& Ceccarelli, C. 2011, A\&A, 532, A39

Maeda, S., \& Ohno, K. 2004a, Chem. Phys. Lett., 398, 240

Maeda, S., \& Ohno, K. 2004b, Chem. Lett., 33, 1372

Maeda, S., \& Ohno, K. 2006, ApJ, 640, 823

Muñoz-Caro, G. M., Meierhenrich, U. J., Schutte, W. A., et al. 2002, Nature, 416, 403

Raghavachari, K., Trucks, G. W., Pople, J. A., \& Head-Gordon, M. 1989, Chem. Phys. Lett., 157, 479

Rimola, A., Sodupe, M., \& Ugliengo, P. 2010, Phys. Chem. Chem. Phys., 12, 5285

Rimola, A., Sodupe, M., \& Ugliengo, P. 2012, ApJ, 754, 24

Snow, J. L., Orlova, G., Blagojevic, V., \& Bohme, D. K. 2007, J. Am. Chem. Soc., 129, 9910

Snyder, LE., Hollis, J. M., Suenram, R. D., et al. 1983, ApJ, 268, 123

Snyder, L. E., Lovas, F. J., Hollis, J. M., et al. 2005, ApJ, 619, 914

Winnewisser, G., \& Churchwell, E. 1975, ApJ, 200, L33

Woon, D. E. 2001, J. Phys. Chem. A, 105, 9478

Woon, D. E. 2002a, ApJ, 571, L177

Woon, D. E. 2002b, Int. J. Quant. Chem., 88, 226

Zuckerman, B., Ball, J. A., \& Gottlieb, C. A. 1971, ApJ, 163, L41 